\title{
Hepatic radiotracer: Still difficult to suppress?
}

\author{
Edward A. Hulten, MD, MPH, FACC, FASNC ${ }^{\mathrm{a}, \mathrm{b}}$ \\ a Cardiology Service, Department of Medicine, Fort Belvoir Community Hospital, Fort Belvoir, \\ VA \\ b Uniformed Services University of Health Sciences, Bethesda, MD
}

Received Feb 14, 2019; accepted Feb 14, 2019

doi: $10.1007 / \mathrm{s} 12350-019-01663-6$

\section{See related article, pp. 2337-2348}

My liver swells with bile difficult to repress. - Horace

Coronary artery disease, CAD, is the leading cause of death and disability in most developed nations and of increasing prevalence in developing nations worldwide. Single-photon emission computed tomography SPECT myocardial perfusion imaging (SPECT MPI), with radiotracer $99 \mathrm{mTc}-m e t h o x y i s o b u t y l i s o n i t r i l e \quad(\mathrm{MIBI})$, provides an accurate, safe, and clinically useful tool for the diagnosis, prognostication, and management of patients with known or suspected CAD. SPECT MPI serves as the most common imaging modality for assessment for possible coronary ischemia with approximately 15-20 million cases annually. ${ }^{1}$ Nevertheless, imaging artifacts may limit SPECT accuracy including motion artifacts, attenuation artifacts from overlying soft tissue structures such as breast, or altered myocardial tracer appearance from excessive bowel or liver tracer.

Whereas ancient physicians sought to repress excessive biliary activity and balance the four humors, modern nuclear cardiologists too seek to suppress hepatic photon emission. In particular, retained tracer uptake in the liver may overlie portions of the heart and could result in Compton scatter that may decrease sensitivity and specificity of the exam both obscuring small regions of diseased myocardium as well as false-positive appearance of perfusion defects.

Reprint requests: Edward A. Hulten, Cardiology Service, Department of Medicine, Fort Belvoir Community Hospital, DeWitt Loop, 9300, Fort Belvoir, VA 22060; edward.a.hulten.mil@mail.mil

J Nucl Cardiol 2020;27:2349-50.

$1071-3581 / \$ 34.00$

Copyright (c) 2019 American Society of Nuclear Cardiology.
In this issue of the Journal, Dr. Sood and colleagues share valuable insight from a small single-center randomized controlled trial of 118 patients clinically referred to SPECT MPI and administered either the choleretic agent ursodeoxycholic acid (UDCA) versus placebo. Their results demonstrate a simple, safe, cheap, and novel method to reduce hepatic MIBI artifacts impacting SPECT MPI, which importantly could improve nuclear cardiology accuracy, reduce the need for confirmatory testing such as invasive or noninvasive angiography, and potentially improve patient outcomes.

SPECT accuracy has improved from initial reports of planar SPECT with Thallium-201 tracer to the present day. In part, this improvement results from not only improved tracer, MIBI compared with Tl-201, but also acquisition protocols, hardware, software post-processing, repositioning, and $\mathrm{CT}$ attenuation correction (AC). Nevertheless, SPECT accuracy remains imperfect with additional need for continued improvement. A recent meta-analysis comparing SPECT MPI to invasive FFR reported a pooled patient-level sensitivity of 0.74 (0.67$0.79)$ and specificity of $0.79(0.74-0.83) .^{2}$ Advancements such as AC can reduce many soft tissue artifacts to improve specificity, but not all sites ${ }^{3}$ employ the technology. As noted in the 2018 ASNC SPECT MPI guideline by Dr. Dorbala et al, artifacts that appear to either increase or decrease myocardial counts result from the common problem of extracardiac tracer activity in bowel or liver, and "There is currently no reliable correction for such artifacts, and attenuation algorithms can even exacerbate them." 4 Similarly, repositioning such as the addition of prone imaging reduces some subdiaphragmatic artifacts especially bowel uptake, but this technique also may prove insufficient or is under-utilized. For example, in the IAEA Nuclear Cardiology Protocols Cross-Sectional Study (INCAPS), Dr. Einstein and colleagues reported significant variability in the use of best practices such as $\mathrm{AC}$ or repositioning, which occurred worldwide at 67 percent of nuclear cardiology laboratories surveyed. ${ }^{3}$ Experienced technicians may 
identify hepatic uptake and simply re-image the patient; however, this too remains an imperfect solution and also may significantly delay workflow. High-resolution cameras, such as Cadmium Zinc Telluride (CZT), improve accuracy, but are similarly under-utilized worldwide and do not completely correct for hepatic MIBI either.

The authors demonstrated in the current study that a short course of $300 \mathrm{mg}$ UDCA administration starting two days prior to SPECT MPI significantly reduced hepatic counts at both 30 and 60 minutes. There was no change or difference in myocardial counts. Accordingly, scan quality was improved in the UDCA pretreated SPECT MPI patients. Indeed, fewer poor quality studies occurred in the UDCA versus placebo group. As noted by the current authors and in the 2018 ASNC SPECT MPI guideline, other methods to reduce extracardiac tracer activity have included delayed or repeat imaging, carbonated beverages, milk, and repositioning but may delay workflow, resulting in variable implementation worldwide. $^{4}$

A simple modification such as UDCA administration could significantly improve SPECT imaging. Thus, the findings of the current RCT do have significance and are of clinical interest. First, any test accuracy process improvement that is so simple, safe, and cheap should be welcomed enthusiastically, if reproducible in larger multicenter studies. Next, reduction of artifacts could one day reduce tracer doses required for diagnostic studies, thereby reducing the overall estimated radiation dose delivered to the patient. Third, reducing artifacts could improve overall sensitivity and specificity that one day might improve outcomes, reduce the need for downstream confirmation either by PET or angiography, and thereby reduce net radiation further. Finally, equivocal SPECT is a common conundrum and considered an appropriate indication for diagnostic clarification via PET $\mathrm{MPI}^{5}$ or coronary angiography. Artifacts attributable to hepatic MIBI likely contribute in part to the NCDR identified high prevalence of normal coronary angiography; ${ }^{6}$ therefore, efforts to improve specificity of the gatekeeper role in noninvasive testing may reduce healthcare costs.

In conclusion, the authors should be congratulated for the successful trial that potentially could offer a simple means for accuracy improvement. From planar to
Anger to SPECT CT and CZT camera, the past, present, and future of SPECT is contingent upon test accuracy. This is increasingly true due to the variety of cardiac diagnostic options available today whether plain exercise testing or advanced imaging such as SPECT MPI, PET, stress echocardiography, stress CMR, CTA, and potentially CT perfusion or FFR-CT. Ideally, the present study results should be reproduced in a multicenter RCT prior to any recommendation for implementation. However, based upon the current study findings, choleretics such as UDCA could prove useful for hepatic MIBI activity difficult to suppress, to paraphrase the ancient Roman poet Horace.

\section{Disclosures}

All the authors declare that there are no financial conflicts of interest. The views expressed in this article are those of the author and do not reflect the official policy of Fort Belvoir Community Hospital, the Defense Health Agency, Department of Defense, or the US Government

\section{References}

1. Einstein AJ. Multiple opportunities to reduce radiation dose from myocardial perfusion imaging. Eur $\mathrm{J}$ Nucl Med Mol Imaging. 2013;40:649-51.

2. Takx RAP, Blomberg BA, Aidi HE, Habets J, de Jong PA, Nagel E, et al. Diagnostic accuracy of stress myocardial perfusion imaging compared to invasive coronary angiography with fractional flow reserve meta-analysis. Circulation. 2015;8:e002666.

3. Einstein AJ, Pascual TNB, Mercuri M, Karthikeyan G, Vitola JV, Mahmarian JJ, et al. Current worldwide nuclear cardiology practices and radiation exposure: results from the 65 country IAEA Nuclear Cardiology Protocols Cross-Sectional Study (INCAPS). Eur Heart J. 2015;36:1689-96.

4. Dorbala S, Ananthasubramaniam K, Armstrong IS, Chareonthaitawee P, DePuey EG, Einstein AJ, et al. Single photon emission computed tomography (SPECT) myocardial perfusion imaging guidelines: instrumentation, acquisition, processing, and interpretation. J Nucl Cardiol. 2018;25:1784-846.

5. Heller GV, Beanlands R, Merlino DA, Travin MI, Calnon DA, Dorbala S, et al. ASNC model coverage policy: Cardiac positron emission tomographic imaging. J Nucl Cardiol. 2013;20:916-47.

6. Patel MR, Peterson ED, Dai D, Brennan JM, Redberg RF, Anderson $\mathrm{HV}$, et al. Low diagnostic yield of elective coronary angiography. N Engl J Med. 2010;362:886-95.

Publisher's Note Springer Nature remains neutral with regard to jurisdictional claims in published maps and institutional affiliations. 